\section{Ernährungsberatung mit Qualität}

m Arbeitskreis Diätetik in der Allergo-

logie e.V. haben sich Diplom-Ökotrophologen und Diätassistenten zusammengeschlossen, die bei Allergien und anderen Nahrungsmittelunverträglichkeiten nach aktuellen, wissenschaftlich abgesicherten Standards beraten. Ein hohes Qualitätsniveau der Beratung ist dabei zukünftig nicht nur Voraussetzung für die Aufnahme in den Arbeitskreis, die Qualitätssicherung durch regelmäßige Fortbildungen im Bereich Allergologie ist jetzt auch in der Satzung verankert. Um aktives Mitglied des Vereins zu bleiben, müssen regelmäßig Fortbildungs- nachweise an eine Anerkennungskommission eingereicht werden. „Dieser Schritt war wichtig, um sich deutlich von den zunehmenden unseriösen und fachlich nicht qualifizierten Ernährungsberatungen bei Allergien und anderen Unverträglichkeiten abzugrenzen", erklärt Dr. Imke Reese, Vorsitzende des Arbeitskreises Diätetik in der Allergologie e.V. „Eine qualitativ hochwertige ernährungstherapeutische Beratung von Patienten mit Nahrungsmittelunverträglichkeiten stellt die Basis für eine gute Zusammenarbeit zwischen Allergologen und Ernährungsfachkraft dar." Beispiele für solche

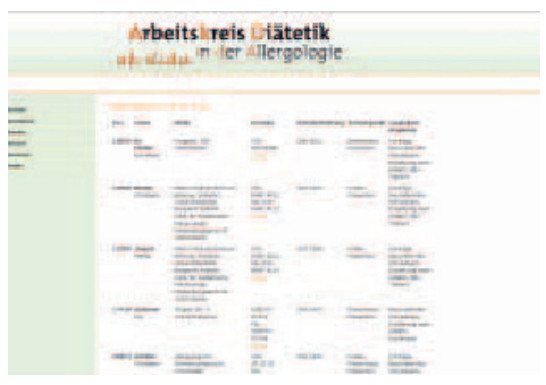

Seit Januar präsentiert sich der Arbeitskreis Diätetik unter www.ak-dida.de neu im Internet. Eine Adressenliste hilft, eine allergologisch versierte Ernährungsfachkraft zu finden.

erfolgreichen Kooperationen seien leider noch zu selten, so Reese.

\title{
Forschungspreis für T-Zell-Forscher
}

m Rahmen der 29. Jahrestagung der Deutschen Gesellschaft für Pädiatrische Pneumologie in München ist im März der internationale KlosterfrauForschungspreis vergeben worden. Der mit $30.000 €$ dotierte Preis geht jährlich an Wissenschaftler, die sich in der Grundlagenforschung, Diagnostik oder Therapie bei kindlichem Asthma bronchiale verdient gemacht haben. Preisträger 2007 ist Dr. Philippe Stock von der Klinik für Pädiatrie mit Schwerpunkt Pneumologie und Immunologie der Charité, Berlin.

Stock wurde für seine Arbeiten zu Funktion und Wirkung von regulatorischen T-Zellen ausgezeichnet. Er entwickelte ein Verfahren, mit dem er regulatorische T-Zellen in vivo oder in vitro erzeugen oder vermehren kann. Er erkannte, dass die in vivo erzeugten, Antigen-induzierten regulatorischen TZellen die humorale Immunantwort der $\mathrm{TH} 2-Z$ ellen hemmen und damit eine Überreaktion der Atemwege verhindern (Stock et al. Nat Immunol 2004, 5: 1149-56). Ein weiteres Experiment erfolgte an zytotoxischen T-Zellen im Mausmodell. Stock fand, dass diese Zellen in Abhängigkeit von der Lokalisation verschiedene Entzündungsmediatoren produzieren. Wahrscheinlich haben sie einen protektiven Effekt auf die
Ausbildung von allergischen Erkrankungen (Stock et al. Eur J Immunol 2004; 34: 1817-27). Der Einfluss natürlicher Killer-T-Zellen auf die Entwicklung von Asthma wurde ebenfalls im Mausmodell untersucht (Akbari et al. Nat Med 2003; 9: 582-8), das Ergebnis: Fehlen natürliche Killer-T-Zellen gibt es keine Überreaktion der Atemwege. Weiterhin erkannte Stock, dass diese Zellen in der Lunge ein in-

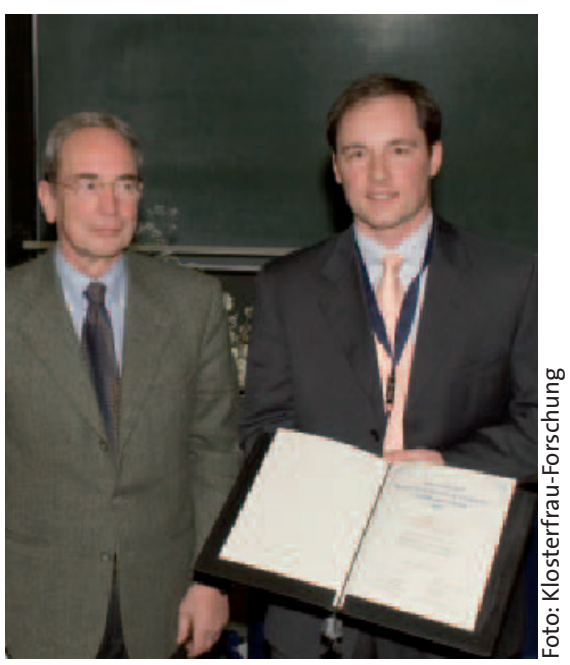

Prof. Dr. Dr. Dietrich Reinhart (links), Kinderklinik München, Vorsitzender der Jury, und der Klosterfrau-Forschungspreisträger 2007 Dr. Phillippe Stock trinsisches Element bereitstellen, das eine humorale Immunantwort der TH2-Zellen erlaubt und dadurch die Überreaktion und Entwicklung von Asthma begünstigt.

Glukokortikoide sind die Basistherapie bei allergischem Asthma, sie hemmen die Effektorfunktion von THelferzellen, Eosinophilen und Epithelzellen. In einer weiteren Arbeit untersuchte Stock die Auswirkungen einer Therapie mit inahalativen Glukokortikoiden auf das Immunsystem (Stock et al. J Immunol 2005; 175: 7380-7). Es zeigte sich, dass unter der Behandlung mit Dexamethason, Prednisolon und Methylprednisolon während eines Allergenkontakts die immunologische Toleranz nicht aufrechterhalten werden konnte. Dadurch kam es zur Sensibilisierung und zu einer heftigen Überreaktion der Atemwege. Glukokortikoide hemmen also auf der einen Seite die akuten Entzündungssymptome, führen aber gleichzeitig zu einer Erkrankungsprogression, da sie die Immunantwort der T-Zellen verstärken. Therapiert werden sollte daher immer mit der geringsten Dosis und in einem engen Zeitfenster. Stock vermutet sogar einen Zusammenhang zwischen dem vermehrten Einsatz von Glukokortikoiden während der vergangenen 20 Jahre und der Zunahme atopischer Ekrankungen im gleichen Zeitraum. 